\section{Smaller and brighter}

The use of super-resolution imaging techniques, such as PALM and STORM, for studying cellular ultrastructures relies on photoactivatable fluorescent proteins (PAFPs). Some characteristics that are key to the performance of these PAFPs include size, brightness, oligomeric state and stability. EosFP is the best overall green-to-red PAFP available,

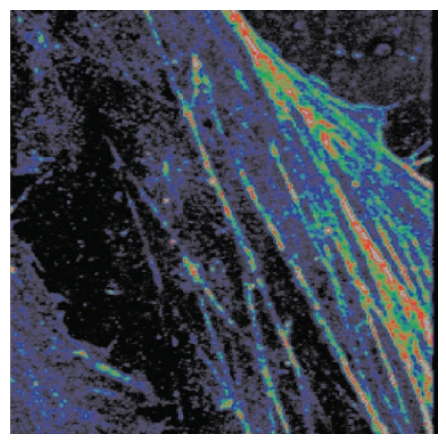
but because it is inherently tetrameric, derivative monomeric and dimeric forms have been generated, with the most widely used being $\mathrm{mEos} 2$. mEos 2 is limited, however, by its tendency to oligomerize at high concentrations, which is worse when it is used to label membrane proteins. Indeed, Pingyong $\mathrm{Xu}$, Tao $\mathrm{Xu}$ and colleagues found that mEos2 fused to Orail, a subunit of the calcium releaseactivated calcium (CRAC) channel, or to glucose transporter 4, causing formation of protein aggregates. In an effort to generate a stable monomeric PAFP, the authors first solved the crystal structure of mEos 2 to determine the molecular basis for its oligomerization. The mEos 2 structure, a tetramer comprised of four $\beta$-cans, revealed three key residues that contribute to oligomerization. Mutation of two of these residues eliminated mEos2's tendency to oligomerize, but the mutant was substantially less bright than the original protein. To enhance brightness, the authors then made additional mutations near the chromophore, resulting in two variants called mEos3.1 and $m E o s 3.2$ that are brighter in the green form than mEos2. Orai1 fusions with mEos3s correctly localized to the plasma membrane and exhibited large CRAC currents, indicating that the fusion interfered minimally with channel function. Additional fusions to other membrane proteins and intracellular markers also showed the expected localization, further demonstrating that the mEos $3 \mathrm{~s}$ behave as good fusion partners. mEos3.2 had the best characteristics overall for green-to-red PAFPs in terms of brightness, stability, monomericity and label density, making it a viable probe for superresolution imaging studies. (Nat. Methods doi:10.1038/nmeth.2021, published online 13 May 2012)

$M M$

\section{Telomere overhang processing}

Telomeres have $3^{\prime}$ single-stranded overhangs that provide substrates for telomerase extension and prevent chromosome ends from being recognized as double-stranded breaks. But how these overhangs are generated and coordinated with the cell cycle has been unknown. Using a combination of biochemical assays, Wright and colleagues now provide insight into the dynamics of telomere overhang formation, revealing an intricate sequence of events. Showing that mature telomere overhangs form within 2 hours of replication in human fibroblast cells, the authors observed that lagging strands have a mature overhang almost immediately after replication. Intriguingly, the final RNA primer of Okazaki-fragment synthesis, which has been thought to be positioned at the very end of the lagging chromosome strand, is instead positioned $70-100$ nucleotides from the end and is not removed for more than 1 hour, defining a specific C-terminal nucleotide. By contrast, leading strands develop a mature overhang only 1-2 hours after replication, and the terminal nucleotide is not specified until the S-to-G2 transition. Together, this suggests that telomere overhang processing occurs in two stages, with the early phase occurring in S phase directly following replication of the duplex telomeric DNA, while the late phase occurs several hours later in the S-to-G2 transition, leaving a considerable delay between the first and second phase. The present work sets the scene for identifying the different factors required for each step and understanding how each step is regulated. (Genes Dev. 26, 1167-1178, 2012) $A H$

\section{The silence of the plants}

Methylation of DNA and dimethylated histone H3 Lys9 (H3K9me2) are marks for epigenetic silencing, leading to higher-order compaction of chromatin. These methylation marks are usually enriched in regions containing transposable elements and DNA repeats, and a deficiency in DNA methyltransferases can result in loss of silencing. Now Jacobsen and colleagues uncover a novel mechanism regulating gene silencing in Arabidopsis thaliana, involving alterations in chromosome superstructure. Using the normally silenced SDC gene with a GFP fusion as a reporter, the authors identified two genes, AtMORC1 and AtMORC6, whose mutations led to loss of SDC silencing. RNA sequencing analyses showed that the derepression effect of the AtMORC gene mutations was also observed for transposable elements and other silenced loci. However, DNA methylation and H3K9me2 levels, as assessed by whole-genome bisulfite sequencing and ChIP-seq respectively, were not affected in atmorc 1 or atmorc 6 mutants. AtMORC 1 and AtMORC6 have close orthologs in higher eukaryotes, and the proteins belong to a broader family of ATPases that includes bacterial DNA gyrase and the mismatch-repair protein MutL. This family had been previously proposed to manipulate DNA superstructures and to remodel chromatin in eukaryotes. Based on this idea, the authors examined chromatin compaction in atmorc 1 and atmorc 6 by microscopy and observed decondensation of the chromocenters, nuclear bodies where pericentromeric heterochromatin usually localizes. They then used $\mathrm{Hi}-\mathrm{C}$ analysis to examine genome-wide chromatin interactions, finding that pericentromeric heterochromatin made many more contacts with the rest of the genome in an atmorc 6 mutant than in the wild type. AtMORC 1 and AtMORC6 proteins seem to localize to the outside of chromocenters. Altogether, these observations led the authors to propose that AtMORC1 and AtMORC6 enforce silencing of pericentromeric heterochromatin by regulating its architecture and promoting its compaction. Interestingly, this silencing mechanism seems to be conserved in worms: the authors showed that knockdown of the single ortholog morc-1 in Caenorhabditis elegans led to derepression of a silenced reporter gene, and previous observations in the mouse knockout are consistent with a role of Morc-1 in silencing of transposable elements during spermatogenesis. (Science 336, 1448-1451, 2012) 